\title{
Coronary recanalization in acute myocardial infarction: immediate results and potential risks
}

\author{
P. W. Serruys, M. van den Brand, T. E. H. Hooghoudt, M. L. Simoons, P. Fioretti, \\ J. Ruiter, P. W. Fels and P. G. Hugenholtz* \\ Thoraxcenter, Erasmus University and University Hospital Dijkzigt, Rotterdam, The Netherlands
}

KEY WORDS: Acute myocardial infarction, recanalization.

Between September 1980 and March 198?, 83 patients were catheterized during the acute phase of their myocardial infarction with the intention to recanalize their infarct-related vessel (IRV); of these 83, 30 participated in a randomized study. Five patients died during the catheterization procedure, two as a result of cardiogenic shock, two of migration of thrombotic material and one of possible heart rupture after a successful recanalization. In 15 patients the IRV was found to be patent at the first coronary injection. In the remaining 64 patients with an occluded IR V, 41 arteries were successfully recanalized.

Of the surviving patients who underwent an attempt at recanalization, 29 had non-fatal complications which required treatment (ventricular fibrillation, ventricular tachycardia or ventricular premature beats, bradycardia, hypotension, artrioventricular block, atrial fibrillation). The complications were predominantly observed in hypotensive patients during angiography after recanalization had been accomplished. Of the 41 successful recanalizations, complications occurred in 20. Of all recanalized right coronary arteries, complications took place in $81 \%$, of the left circumflex arteries in $25 \%$ and of the left anterior descending arteries in $24 \%$.

In conclusion, catheterization and attempts at recanalization of occluded arteries impose a substantial risk of fatal and non-fatal complications. These occur in particular during the first angiogram after the re-opening of an occluded right coronary artery. These observations lead to the following recommendations:

(1) streptokinase infusion should not be considered before the circulation is adequately supported;

(2) lidocaine and nifedipine should be administered prophylactically before the attempt at recanalization;

(3) a non-ionic contrast medium should be used for angiography.

In May 1981 Muller et al. published an editorial on the problems related to coronary recanalization in the acute phase of myocardial infarction with the thought-provoking title: 'Let's not let the genie escape from the bottle, again'[1]. In spite of this warning we are presently confronted with the possibility of premature dissemination of this new technique for treatment of ischemic heart disease, before scientific proof of its beneficial effects has

Received for publication 24 May 1982.

Requests for reprints to: P. W. Serruys, Catheterization Laboratory, Erasmus University and University Hospital Dijkzigt. Dr Molewaterplein 40, 3000 DR Rotterdam. The Netherlands.

*With the technical assistance of S. Veldhof-Cocks. been demonstrated and before adequate knowledge has been acquired about the potential risks of the procedures. Before this intervention can be recommended for general use, many problems should be solved ${ }^{[2]}$. In this paper, based on the experience with 83 patients in whom intracoronary thrombolysis was attempted at the Thoraxcenter, tentative answers are formulated on two questions: what time delay can be accepted between the onset of symptoms of myocardial infarction and attempted recanalization and what are the potential dangers of intracoronary fibrinolysis?

\section{Patient selection and methods}

Since our first experience with intracoronary thrombolysis in September 1980, different proto- 
Table 1 Study population September 1980-March 1982. Numbers between brackets are additional patients who refused angiography

\begin{tabular}{|c|c|c|c|c|c|c|}
\hline \multirow{2}{*}{$\overbrace{\begin{array}{l}\text { Strepto- Uro- } \\
\text { kinase kinase }\end{array}}^{\text {Pilot study } 1}$} & \multicolumn{3}{|c|}{ Pilot study 2} & \multirow{3}{*}{$\begin{array}{l}\text { Pilot study } 3 \\
\text { Streptokinase }\end{array}$} & \multicolumn{2}{|c|}{ Randomized study } \\
\hline & & & Control & & Strepto- & Control \\
\hline & $\begin{array}{c}\text { Uro } \\
\text { kinase } \\
\text { i.c. }\end{array}$ & $\begin{array}{l}\text { Nife- } \\
\text { dipine } \\
\text { i.v. }\end{array}$ & & & & \\
\hline
\end{tabular}

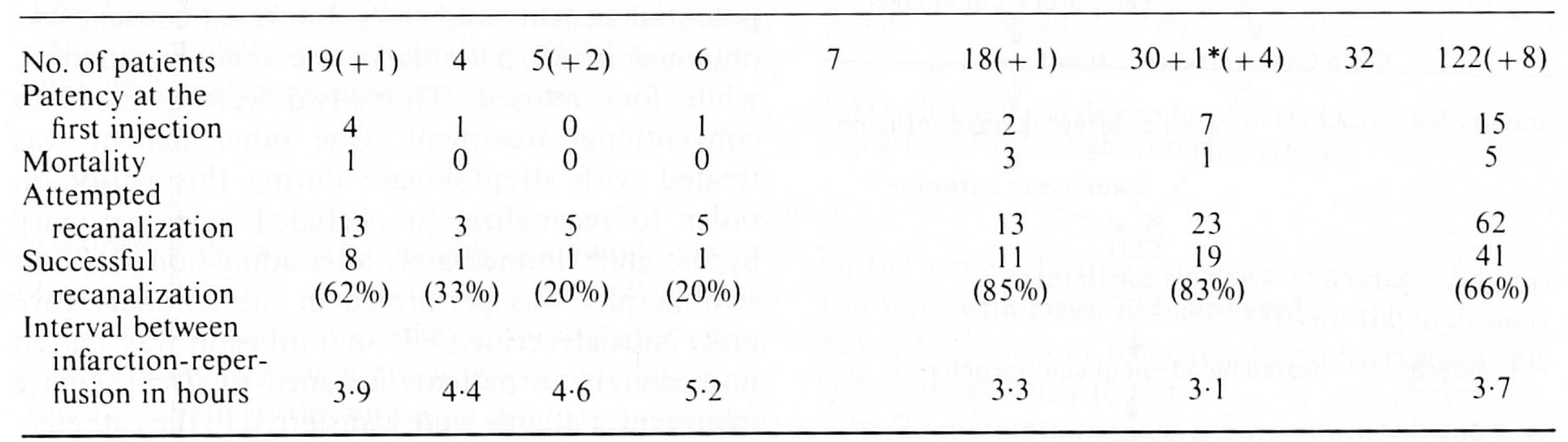

${ }^{*}$ One patient was not randomized.

cols have been used. These are described in this section, with the reasons which prompted us to adapt the procedures. In retrospect our initial experience can be discerned as three pilot studies (Table 1). The protocol of the present randomized trial is described in detail in Fig. 1.

PILOT STUDY 1 (23 PATIENTS - SEPTEMBER 1980 UNTIL NOVEMBER 1980)

In this first phase, the procedure was started with ventriculography in the right anterior oblique position and complete coronary angiography, beginning with the artery suspected not to be thrombosed. A brachial arteriotomy (Sones) was used since it was our belief that this would provide better control of hemostasis than the percutaneous technique. Urographin $76 \%$ served as the contrast medium. Before intracoronary fibrinolysis, $0.15 \mathrm{mg}$ nitroglycerine and $0.1 \mathrm{mg}$ nifedipine, both in an alcoholic solution, were injected directly into the coronary artery to exclude spasm as the cause of obstruction. Streptokinase perfusion was then started at a rate of 2000 units/min with a maximum dosage of 250000 units. Thereafter ventriculography was repeated. In the last four patients streptokinase was replaced by urokinase at the same dosage, since it was expected that streptokinase would cause allergic reactions in some of the patients. In the first pilot study, one patient refused the procedure when his consent was asked.
PILOT STUDY 2 (18 PATIENTS - NOVEMBER 1980 UNTIL MARCH 1981)

The second phase was characterized by an effort to compare three randomly allocated groups of patients: patients treated with intracoronary fibrinolysis with urokinase as described above, patients in whom solely intracoronary nitroglycerine and intravenous nifedipine at a dosage of $1 \mathrm{mg}$ in $5 \mathrm{~min}$ would be administered, and a control group of patients treated by conventional methods in the coronary care unit. In order to study myocardial perfusion and reperfusion, thallium scintigraphy was performed at admission and after $4 \mathrm{~h}$. After the inclusion of 18 patients it was decided to interrupt this study since thrombolysis was achieved in only one out of five patients treated with urokinase and since the requirements of the protocol lead to an excessive delay between admission and initiation of therapy. Two additional patients refused angiography in the acute phase of myocardial infarction.

PILOT STUDY 3 (18 PATIENTS - MARCH 1981 UNTIL JUNE 1981)

After this negative experience, it was decided to simplify the procedure and to reduce the delay between the onset of symptoms and initiation of fibrinolysis. The percutaneous femoral approach after Judkins was used instead of the Sones method. Left ventricular angiograms were abandoned, and no attempt was made to visualize the artery not sus- 


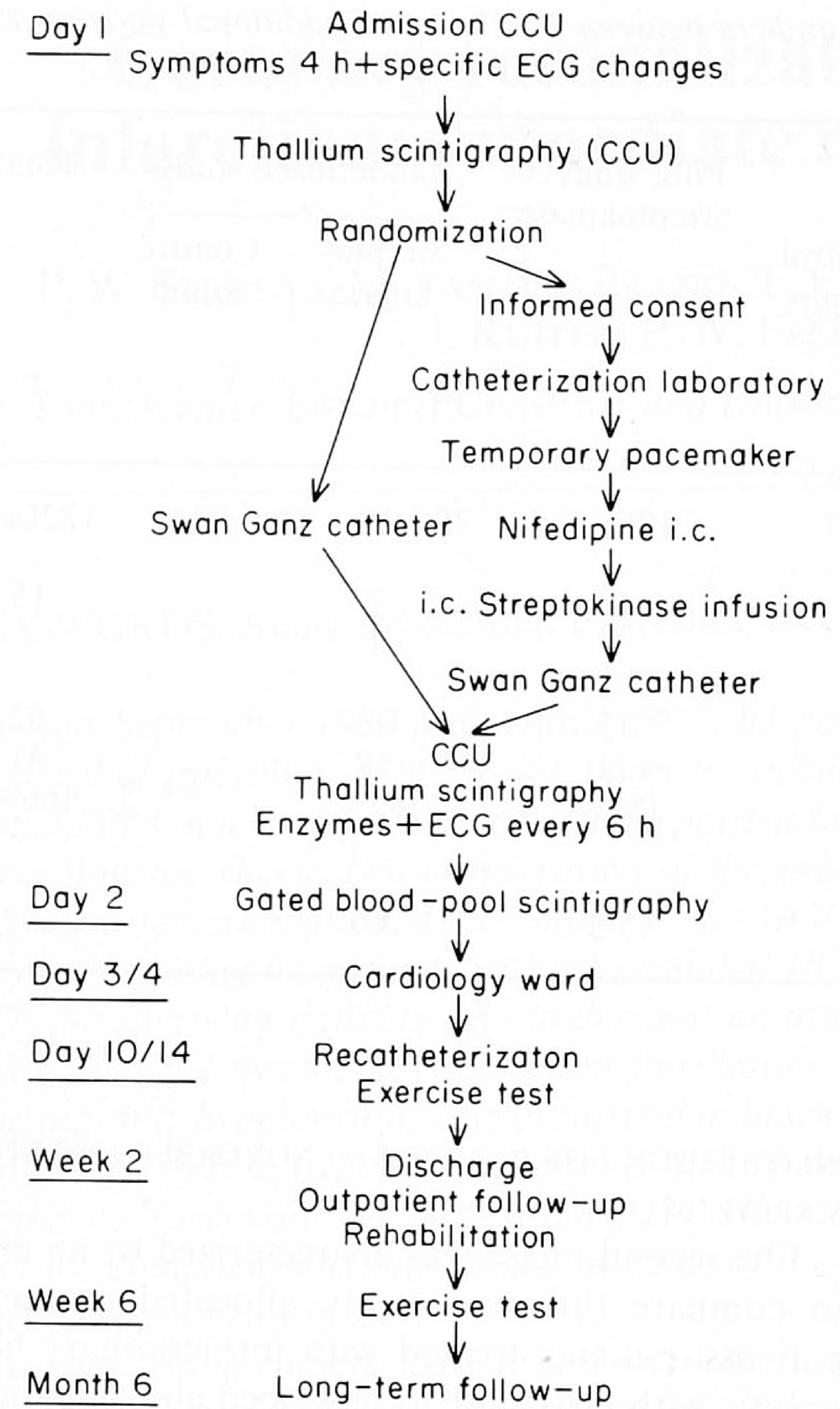

Figure 1 Flow chart of the randomized trial: intracoronary streptokinase $v$. conventional treatment in myocardial infarction.

pected to be thrombosed. Streptokinase was used again, and the dosage was increased to 4000 units/ min. In these 18 patients satisfactory results were obtained. One patient refused to give his consent.

RANDOMIZED TRIAL (66 PATIENTS - JUNE 1981 UNTIL MARCH 1982 AND CONTINUING)

In this randomized study the selection criteria were more strict than before. Patients were selected below 65 years of age, without a history of hemorrhagic diathesis or previous cerebral vascular accident. The delay between the onset of symptoms and admission to the study had to be less than $4 \mathrm{~h}$. The electrocardiogram had to show ST segment elevation of $0.2 \mathrm{mV}$ or greater in the precordial leads, or $0.1 \mathrm{mV}$ or more in the standard leads. Chest pain had to be present on admission and could subsequently be treated with thalamonal or opiates if required, before streptokinase intervention was initiated. The combination of hypotension (systolic pressure below $90 \mathrm{mmHg}$ ) and sinus tachycardia (heart rate over 100 beats/min) led to temporary exclusion. If the hemodynamic condition of the patient recovered quickly he or she could still be included in the study. The flow chart is provided in Fig. 1.

As in the pilot studies, all patients assigned to attempted thrombolysis and their relatives, when present, were informed about the procedure, its potential benefits and risks. Informed consent was obtained in 30 patients in the randomized trial, while four refused. Thirty-two were assigned to conventional treatment. One other patient was treated with streptokinase during this period in order to recanalize an occluded aorto-coronary bypass graft. Immediately after admission thallium scintigraphy was performed in the coronary care unit. Nitroglycerine $(0.15 \mathrm{mg})$ infusion was started immediately in patients assigned to streptokinase treatment. Patients were transferred to the catheterization laboratory as soon as possible and lidocaine was given intravenously in a dose of $2 \mathrm{mg} / \mathrm{min}$. After puncturing the femoral vein and artery a pacemaker catheter was positioned in the right ventricle. Next, coronary arteriography of the artery suspected to be thrombosed was performed. A non-ionic contrast agent (Amipaque ${ }^{R}$ ) was employed as contrast medium. After identification of the thrombosed coronary branch, $50 \mathrm{mg}$ heparin was administered intravenously together with $250 \mathrm{mg}$ acetylsalicylic acid and $100 \mathrm{mg}$ diadresone $\mathrm{F}^{\mathrm{R}}$, a corticosteroid. Before starting the intracoronary perfusion with streptokinase, $0.2 \mathrm{mg}$ nifedipine was injected into the thrombosed coronary artery within $3 \mathrm{~min}$, while the aortic pressure was monitored. Coronary arteriography was then repeated to evaluate the spasmolytic effect on the coronary occlusion. Intracoronary perfusion with streptokinase was carried out at a rate of 4000 units $/ \mathrm{min}$ to a maximum of 250000 units of streptokinase, diluted in $500 \mathrm{ml}$ of physiological solution at a flow rate of $8 \mathrm{ml} / \mathrm{min}$. Coronary angiograms were repeated every 15 minutes until the chest pain disappeared. The appearance of ventricular extrasystoles or any other conduction disturbance was also a reason to revisualize the artery. If there were no signs of recanalization, an attempt was made to administer streptokinase locally in a higher concentration by passing a thin catheter (French 2) with a radiopaque tip through the Judkins catheter (French 8). After the procedure, a left ventriculogram was made with $0.75 \mathrm{ml}$ contrast medium $/ \mathrm{kg}$ body weight, provided LVEDP did not exceed 30 
$\mathrm{mm} \mathrm{Hg}$. The patient was transferred to the coronary care unit where blood samples were analyzed every $6 \mathrm{~h}$ to determine the creatine phosphokinase (CPK) levels and general supportive therapy was provided. The day before discharge from the intermediary care area, coronary arteriography and left ventricular arteriography were repeated, usually during the third week after infarction. About that time the patients also underwent a maximal exercise test on a bicycle ergometer to evaluate their exercise capacity, any recurrence of angina pectoris and electrocardiographic changes, if any.

\section{Results}

ANGIOGRAPHIC RESULTS OF ATTEMPTED

RECANALIZATION

In 15 patients $(18 \%)$, the infarct-related vessel was found to be open at the first coronary injection. In one patient in the first pilot study, an occluded postero-lateral branch of the right coronary artery was overlooked at angiography. No streptokinase was administered since the angiogram was falsely interpreted as being completely normal. Excluding the four deaths, 62 patients have undergone an attempt at recanalization, 49 of whom received intracoronary streptokinase, eight intracoronary urokinase and five intravenous nifedipine.

The percentage of success and failure of recanalization with regard to the coronary anatomy and the drug administered is detailed in Table 2. The percentage of successful recanalizations are, respectively, $77 \%$ for streptokinase, $25 \%(2 / 8)$ for urokinase and $20 \%(1 / 5)$ for intravenous nifedipine (Table 2).

DELAY BETWEEN THE ONSET OF SYMPTOMS AND THE BEGINNING OF INTRACORONARY FIBRINOLYSIS: DOES IT INFLUENCE THE DIRECT RESULTS?

During both the first pilot study and the first randomized study, patients were admitted for intracoronary fibrinolysis until $8 \mathrm{~h}$ after the beginning of the symptoms, provided that they were still symptomatic. During the first pilot study, the delay ranged from 80 to $495 \mathrm{~min}$ with a mean of $247 \mathrm{~min}$ (s.d. $146 \mathrm{~min}$ ). In the second phase the delay was even longer (mean $288 \mathrm{~min}$, s.d. $80 \mathrm{~min}$ ), ranging from 135 to $405 \mathrm{~min}$. After these first two experiences, a maximal time limit of $4 \mathrm{~h}$ was imposed. In consequence the delay was diminished during the third phase of the study to a mean of $198 \mathrm{~min}$ (s.d. $53 \mathrm{~min}$ ), ranging from 95 to $285 \mathrm{~min}$. Currently, during the ongoing randomized trial based
Table 2 Percentage of successful recanalizations with respect to the anatomical site of the obstruction and applied pharmacological intervention

Streptokinase Urokinase Nifedipine i.v.

\begin{tabular}{lcrcl}
\hline LAD & $15 / 23$ & $65 \%$ & $1 / 6$ & $1 / 2$ \\
LCX & $8 / 8$ & $100 \%$ & - & $0 / 2$ \\
RCA & $15 / 18$ & $83 \%$ & $1 / 2$ & $0 / 1$ \\
Total & $38 / 49$ & $77 \%$ & & \\
\hline
\end{tabular}

$\mathrm{LAD}=$ left anterior descending artery; $\mathrm{LCX}=$ left circumflex artery; RCA = right coronary artery.

on the same selection criteria, the average delay is $186 \mathrm{~min}$, which can possibly explain the high incidence of success of recanalization observed in this last phase of study $(83 \%)$

In 25 patients (before a time limit of $4 \mathrm{~h}$ was imposed) the relationship between the time of the onset of symptoms, the delay before admission to the catheterization laboratory and the duration of intracoronary perfusion of a fibrinolytic enzyme (streptokinase) could be determined precisely (Fig. 2 ). Three conclusions can be drawn from our initial experience:

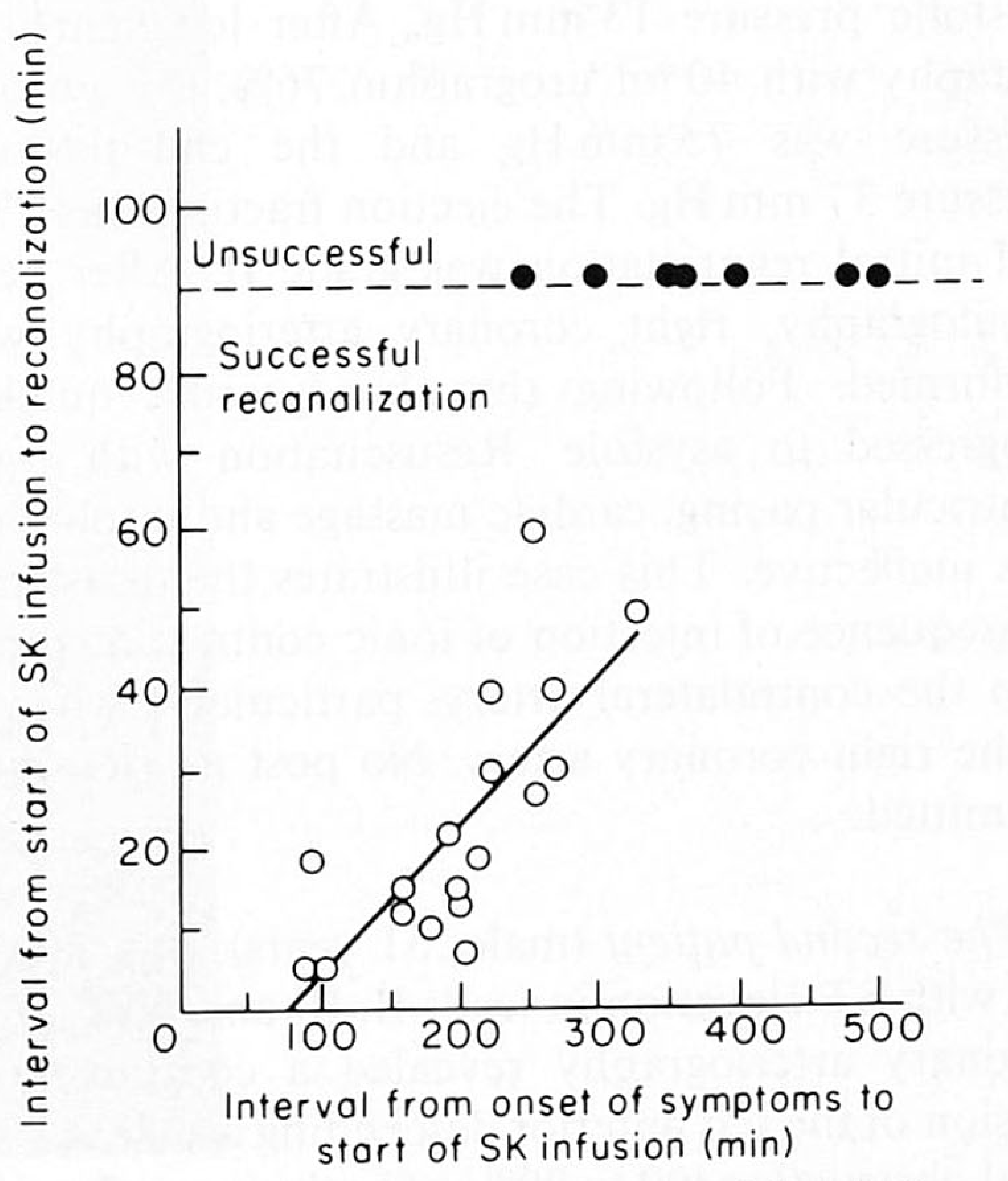

Figure 2 Relationship between the delay before admission to the catheterization laboratory and the time interval between the onset of fibrinolysis and the first angiographic opacification of the coronary branch distal to the thrombotic lesion. $y=-14+b x, r=0 \cdot 76$. $\mathrm{SK}=$ streptokinase. 
(1) after $6 \mathrm{~h}$, success of recanalization is nil;

(2) before the 4 th $\mathrm{h}$ the great majority of the obstructed arteries can be recanalized by intracoronary fibrinolysis;

(3) in the group of patients with successful recanalization a relationship exists between the delay before admission to the catheterization laboratory and the time interval between the onset of fibrinolysis and the first angiographic opacification of the coronary branch distal to the thrombotic lesion.

\section{COMPLICATIONS DURING THE PROCEDURE}

\section{Fatal complications}

Of the 83 patients that were catheterized during the acute phase of myocardial infarction with the purpose of recanalization, five died during the catheterization procedure, three before fibrinolysis could be started, one during the fibrinolysis and one after a successful fibrinolysis. This high percentage of mortality during the catheterization necessitates the individual analysis of the 5 deaths.

The first patient (male, 32 years) presented with an antero-septal and antero-lateral infarction. On admission, a sinus tachycardia of 110 beats/ min was present. He was hypotensive and in shock. During the catheterization the left ventricular systolic pressure was $69 \mathrm{~mm} \mathrm{Hg}$ and the enddiastolic pressure $13 \mathrm{~mm} \mathrm{Hg}$. After left ventriculography with $40 \mathrm{ml}$ urographin $76 \%$, the systolic pressure was $75 \mathrm{~mm} \mathrm{Hg}$ and the end-diastolic pressure $37 \mathrm{~mm} \mathrm{Hg}$. The ejection fraction was $27 \%$ and mitral regurgitation was grade II. After ventriculography, right coronary arteriography was performed. Following this, bradycardia quickly progressed to asystole. Resuscitation with right ventricular pacing, cardiac massage and intubation was ineffective. This case illustrates the disastrous consequence of injection of ionic contrast medium into the contralateral artery, particularly when it is the right coronary artery. No post mortem was permitted.

The second patient (male, 51 years), was admitted with ST elevation in leads II, III and AVF. The coronary arteriography revealed a complete occlusion of the left anterior descending artery, a subtotal obstruction (90 to $99 \%$ ) of both circumflex and right coronary arteries (90 to 99\%). During catheterization, ST elevation disappeared together with the precordial pain so that intracoronary fibrinolysis was not performed. Four days after the first catheterization the patient presented again with chest pain, accompanied by ST elevation in leads II, III and AVF. This time, right coronary arteriography showed a total obstruction of this vessel. During perfusion with streptokinase, the systolic blood pressure fell to $70 \mathrm{~mm} \mathrm{Hg}$; this was soon followed by complete atrioventricular block and electromechanical dissociation despite right ventricular pacing. No post mortem was performed. In this case the deplorable state of the coronary vessels, the proximal localization of the lesions as well as the dominant character of the right coronary artery should have served as indicators for a subsequent fatal outcome once total obstruction of this coronary artery had taken place.

The third patient (male, 41 years) was admitted because of an anterior wall infarction. The first arteriogram in the right anterior oblique projection (Judkins catheter), demonstrated a very short main stem, a total occlusion of the left anterior descending artery proximal to the septal branches and less than $50 \%$ obstruction of the circumflex artery; $0.2 \mathrm{mg}$ nifedipine and $0.15 \mathrm{mg}$ nitroglycerin were injected into the main stem artery (Fig,. 3). Three minutes later, a control coronary angiogram demonstrated extremely slow progression of the contrast medium and absence of visualization of the distal part of the circumflex artery. Shortly thereafter, a new injection showed a total occlusion of the circumflex artery. Almost immediately the patient developed severe hypotension and asystole. At post mortem, a loose thrombus was found unattached to the wall in the circumflex artery. In this patient, it is suspected that the tip of the Judkins catheter dislocated the proximal part of the thrombus situated in the left anterior descending artery so that it could have obstructed the left circumflex artery as an embolus.

The fourth patient experienced the same lethal accident as the previous case and the coronary anatomy was quite similar. During the first contrast injection, the Judkins catheter lost its selective position in the main stem artery, so that it had to be repositioned. During the second contrast injection stagnation of the contrast medium in the circumflex artery was observed with a total obstruction of the distal part. No post mortem was performed.

The fifth patient (male, 58 years), was admitted with an infero-lateral myocardial infarction. Ten minutes after infusion of streptokinase into the occluded right coronary artery, distal filling of 

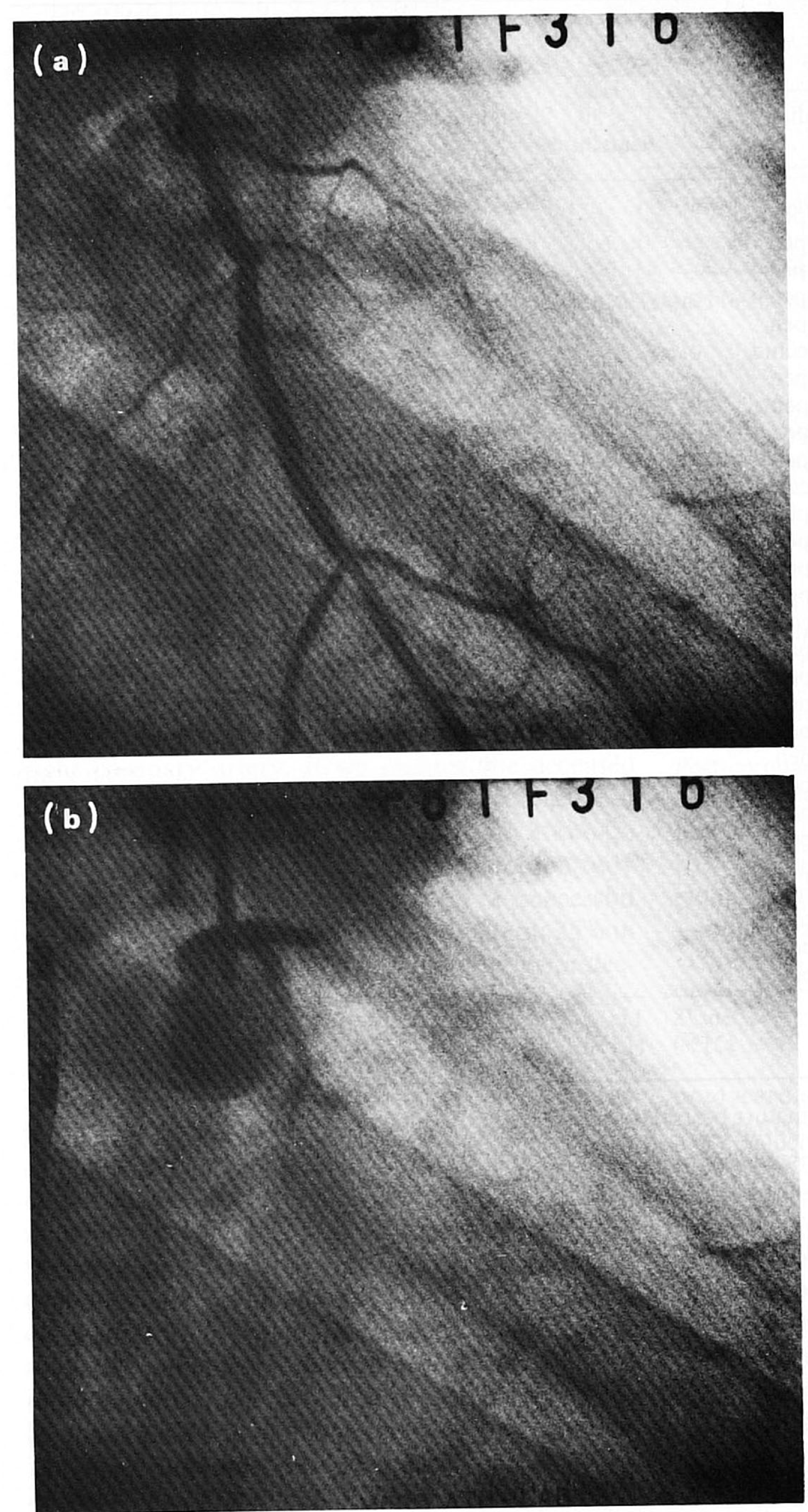

Figure 3 Sequential left coronary arteriograms in the right anterior oblique projection, demonstrating a proximal occlusion of the left anterior descending artery and subsequently the acute occlusion of the left circumflex artery. 
Table 3. Non-fatal complications during attempted recanalization ( $n=78$ patients)

\begin{tabular}{|c|c|c|c|c|c|c|c|c|c|c|c|}
\hline \multirow[b]{2}{*}{$\begin{array}{c}\text { Patient } \\
\text { No. }\end{array}$} & \multirow{2}{*}{$\begin{array}{c}\text { Delay } \\
\text { onset of } \\
\text { symptoms } \\
\text { fibrinoloysis } \\
(\mathrm{min})\end{array}$} & \multirow[b]{2}{*}{$\mathrm{CA}$} & \multicolumn{2}{|c|}{ Status } & \multirow[b]{2}{*}{ VPB } & \multirow[b]{2}{*}{$\begin{array}{c}\mathrm{HR} \\
<50 \\
\text { beats/min }\end{array}$} & \multirow[b]{2}{*}{$\begin{array}{c}\mathrm{BP} \\
<90 \mathrm{~mm} \mathrm{Hg}\end{array}$} & \multirow[b]{2}{*}{$\overbrace{\mathrm{DCCS}(\mathrm{x})}^{\mathrm{VF}}$} & \multirow[b]{2}{*}{$\begin{array}{c}\text { A-V } \\
\text { block }\end{array}$} & \multirow[b]{2}{*}{ VT } & \multirow[b]{2}{*}{$\mathrm{AF}$} \\
\hline & & & $\overbrace{\text { before }}$ & sis & & & & & & & \\
\hline $1 \mathrm{D}$ & - & $\mathrm{RCA}$ & Open & Open & & o & & & & & \\
\hline $2 \mathrm{~K}$ & 225 & RCA & Occlud. & Open & & o & o & & & & \\
\hline $3 \mathrm{R}$ & 180 & LAD & Open & Open & & & & & & & o \\
\hline $4 \mathrm{~K}$ & 240 & $\mathrm{RCA}$ & Occlud. & Open & $\mathrm{O}$ & & & $O(3 x)$ & o & & o \\
\hline $5 \mathrm{VE}$ & 120 & LCX & Occlud. & Open & & & & & & o & \\
\hline $6 \mathrm{DG}$ & 495 & LAD & Occlud. & Occlud. & o & & o & & & & \\
\hline $7 \mathrm{Sch}$ & 270 & RCA & Occlud & Open & 0 & o & & & & & \\
\hline $8 \mathrm{~B}$ & 435 & RCA & Open & Open & o & o & & & o & $\mathrm{o}(5 \mathrm{x})$ & $\mathrm{o}$ \\
\hline $9 \mathrm{R}$ & 285 & RCA & Occlud. & Open & o & 0 & o & $\mathrm{o}(4 \mathrm{x})$ & & 0 & 0 \\
\hline $10 \mathrm{Sch}$ & 225 & RCA & Occlud. & Open & & o & & o $(26 x)$ & $\mathrm{o}$ & & $\mathrm{o}$ \\
\hline $11 \mathrm{~K}$ & 240 & LAD & Occlud. & Occlud. & $\mathrm{o}$ & & & & & $\mathrm{O}$ & \\
\hline $12 \mathrm{H}$ & 165 & RCA & Occlud. & Open & o & o & & $O(1 x)$ & o & o & \\
\hline $13 \mathrm{VS}$ & 255 & RCA & Occlud. & Open & o & o & o & $o(1 x)$ & o & & o \\
\hline $14 \mathrm{~K}$ & 210 & RCA & Occlud. & Open & 0 & & 0 & & o & & \\
\hline $15 \mathrm{VH}$ & 195 & RCA & Occlud. & Open & $\mathrm{o}$ & & & & & & \\
\hline $16 \mathrm{~F}$ & 240 & RCA & Occlud. & Occlud. & & & o & & & & \\
\hline $17 \mathrm{ST}$ & 255 & RCA & Occlud. & Open & & & & $0(5 x)$ & o & & \\
\hline $18 \mathrm{D}$ & 195 & LAD & Occlud. & Open & o & & & $o(1 x)$ & & & \\
\hline $19 \mathrm{DC}$ & 150 & LAD & Occlud. & Open & & o & o & & & & \\
\hline $20 \mathrm{~K}$ & 210 & RCA & Occlud. & Open & o & & & $o(1 x)$ & & o & \\
\hline $21 \mathrm{R}$ & 165 & RCA & Occlud. & Open & & & $\mathrm{o}$ & & & & \\
\hline $22 \mathrm{~L}$ & 135 & LCX & Occlud. & Open & & & o & & 0 & o & \\
\hline $23 \mathrm{~S}$ & - & RCA & Occlud. & Open & o & 0 & $\mathrm{O}$ & & & & \\
\hline $24 \mathrm{~B}$ & 132 & RCA & Occlud. & Open & & o & o & & & & \\
\hline $25 \mathrm{~S}$ & 315 & LAD & Occlud. & Open & o & & & & & & \\
\hline $26 \mathrm{DA}$ & 215 & LAD & Occlud. & Occlud. & o & & & & & & \\
\hline $27 \mathrm{DAA}$ & 198 & $\mathrm{RCA}$ & Occlud. & Occlud. & & & & $\mathrm{o}(2 \mathrm{x})$ & & & \\
\hline \multirow{3}{*}{$29 \mathrm{D}$} & 265 & LAD & Occlud. & Open & o & & & & & & \\
\hline & 130 & $\mathrm{RCA}$ & Occlud. & Open & & & $\mathrm{o}$ & $o(1 x)$ & & & \\
\hline & & & & & $\begin{array}{l}16 / 78 \\
(21 \%)\end{array}$ & $\begin{array}{l}11 / 78 \\
(15 \%)\end{array}$ & $\begin{array}{l}12 / 78 \\
(16 \%)\end{array}$ & $\begin{array}{l}10 / 78 \\
(13 \%)\end{array}$ & $\begin{array}{c}8 / 78 \\
(11 \%)\end{array}$ & $\begin{array}{l}7 / 78 \\
(9 \%)\end{array}$ & $\begin{array}{l}6 / 78 \\
(7 \%)\end{array}$ \\
\hline
\end{tabular}

$\mathrm{CA}=$ coronary artery; $\mathrm{VPB}=$ ventricular premature beats; $\mathrm{HR}=$ heart rate; $\mathrm{BP}=$ blood pressure; $\mathrm{VF}=$ ventricular fibrillation; $\mathrm{CS}=$ counter shock; $\mathrm{A}-\mathrm{V}=$ atrioventricular; $\mathrm{VT}=$ ventricular tachycardia; $\mathrm{AF}=$ atrial fibrillation.

the vessel was observed. Thirty minutes after successful recanalization his blood pressure suddenly dropped to $30 \mathrm{~mm} \mathrm{Hg}$, despite normal sinus rhythm. Despite the administration of positive inotropic drugs and volume expanders, no restoration of the blood pressure was observed. No post mortem was performed. Cardiac rupture is suspected.

\section{Non-fatal complications}

A description of the complications that necessitated therapeutic intervention (antiarrhythmic drug administration, pacemaker therapy, inotropic drug administration, plasma, etc.) is summarized in
Table 3. Unifocal and multifocal extrasystoles, doublets and bigeminy are the most common $(21 \%)$ rhythm disturbances encountered during the procedure. Less frequent are hypotension (blood pressure lower than $90 \mathrm{~mm} \mathrm{Hg}$ ) in $16 \%$ of the patients, bradycardia (heart rate less than 50 beats/min) in $15 \%$ of the patients, ventricular fibrillation in $13 \%$ of the patients, atrioventricular block in $11 \%$ of the patients, ventricular tachycardia (three or more ventricular beats) in $9 \%$ and atrial fibrillation or flutter in $7 \%$ of the patients.

It is noteworthy that when ventricular fibrillation occurred in an individual patient, it was frequently repetitive so that in 10 patients a total of 45 defib- 


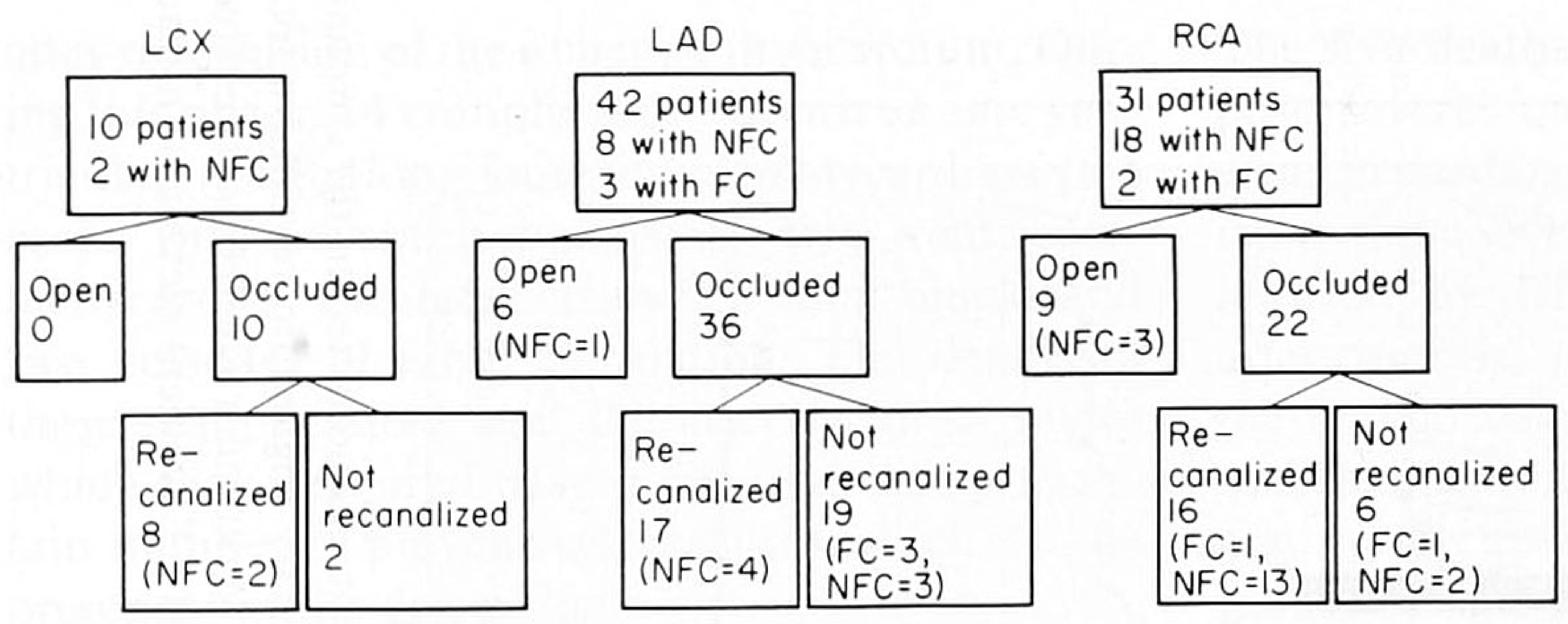

Figure 4 Fatal (FC) and non-fatal (NFC) complications related to the site of coronary obstruction and result of recanalization.

rillations were performed. When analysing these complications, it must be emphasized that the incidence of complications is particularly high in those patients where an attempt to reperfuse the right coronary artery was undertaken. Indeed, three groups of patients can be distinguished: 10 patients with suspected thrombosis of the circumflex artery, 42 patients with suspected thrombosis of the left anterior descending artery or one of its branches and 31 patients with suspected thrombosis of the right coronary artery. If we include the deceased patients, the percentage of complications is $20 \%$ in the first group, $26 \%$ in the second group and $64 \%$ in the third group. Taking into consideration only the non-fatal complications during a successful recanalization, the percentages are different: $25 \%$ in the circumflex artery, $24 \%$ in the left anterior descending artery and $81 \%$ in the right coronary artery and its branches (Fig. 4). This last group is thus characterized by a very high incidence of complications such as ventricular arrhythmias, hypotension and atrioventricular block (Fig. 5).

The stages in the procedure during which these complications occurred are presented in Table 4 . A certain number of complications are inherent in the acute phase of a myocardial infarction. Fifteen events occurred spontaneously in the catheterization laboratory before any intervention or manipulation of the catheter within the heart. In our initial experience we proceeded with left ventriculography before performing coronary angiography. This diagnostic procedure was carried out for investigative purposes and was related to two ventricular fibrillations, one sinus bradycardia, one severe hypotension, one ventricular tachycardia, one atrioventricular block and one atrial fibrillation. Also the first diagnostic coronary angiogram before recanalization of the vessel is not without risk, since one ventricular fibrillation, one
Table 4 Occurrence of non-fatal complications $(n=70)$ during the different stages of the procedure

Percentage

During fibrinolysis

$26 \% \quad(18 / 70)$

Occurring in the catheterization

laboratory before catheterization $\quad 21 \% \quad(15 / 70)$

Post-coronary angiogram after recanalization $\quad 20 \% \quad(14 / 70)$

Post-LV angiogram $\quad 10 \% \quad(7 / 70)$

Post-coronary angiogram before recanalization

Post-cardioversion

Post-nitroglycerin i.c.

Post-nifedipine i.c.

During right heart catheterization

$7 \% \quad(5 / 70)$

$6 \% \quad(4 / 70)$

$4 \% \quad(3 / 70)$

$3 \% \quad(2 / 70)$

$3 \% \quad(2 / 70)$

$100 \% \quad 70$

bradycardia, one hypotension and one total atrioventricular block were observed. The following step involved intracoronary injection of nitroglycerin and/or nifedipine. In five cases this led to bradycardia or hypotension and in one patient in whom the vessel was opened under the influence of intracoronary nifedipine, ventricular fibrillation occurred. In this case reperfusion was very fast and complete.

The percentage of complications observed during the perfusion of the fibrinolytic agent was $26 \%$; the rhythm disturbances observed were mainly uniform and multiform ventricular premature complexes, sometimes associated with doublets and bigeminy. In four cases, severe arterial hypotension developed during the perfusion with streptokinase and in two patients bradycardia was observed. The most critical step of the recanalization procedure in our experience is coronary arteriography immediately 


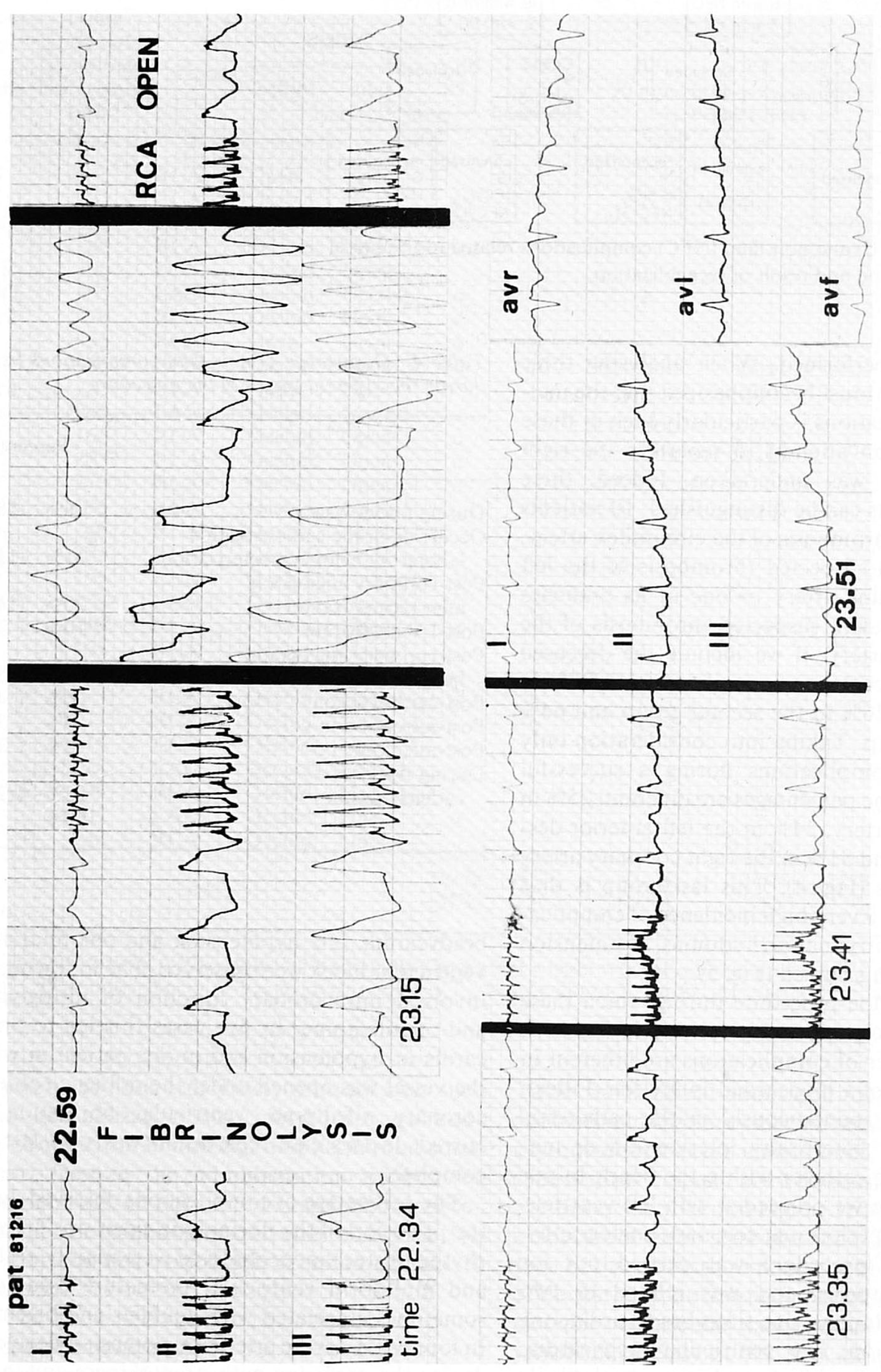

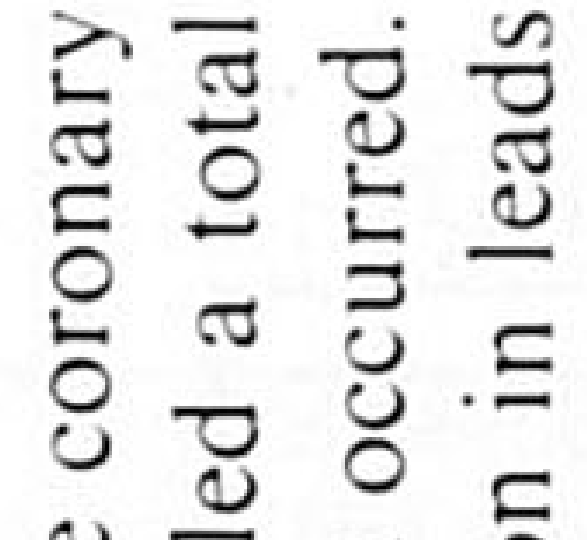

Ð

인 氜

¿

ह

동

츤

$3 \geq 2$

苛要次

हैं

记

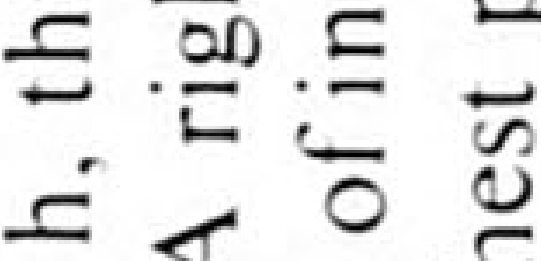

प

证的

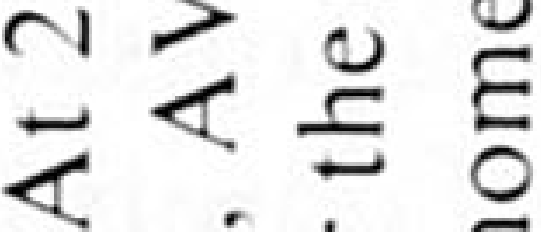

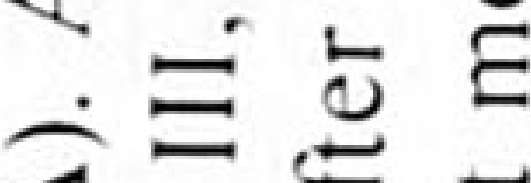

$\overline{0}=0$

$\cong$ 正

它导㤩

可 $\cong$

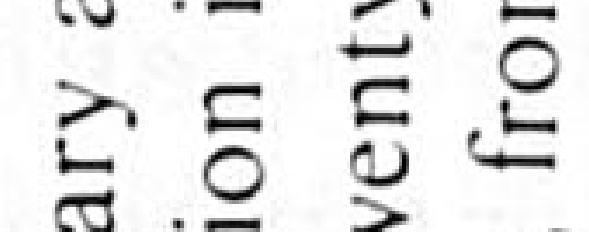

윤

ठ․ ए

志昰志

$\approx$ के थै

क 3

ప氙这

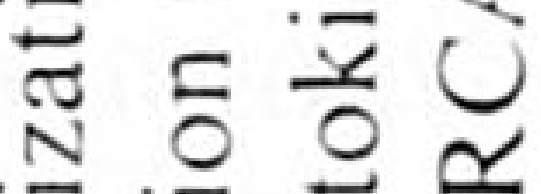

든 인

过

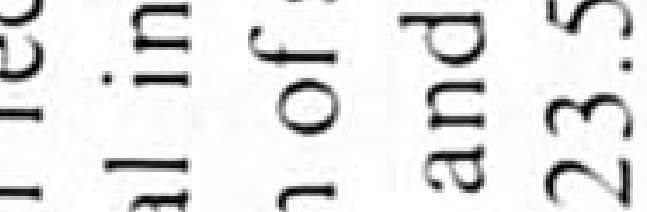

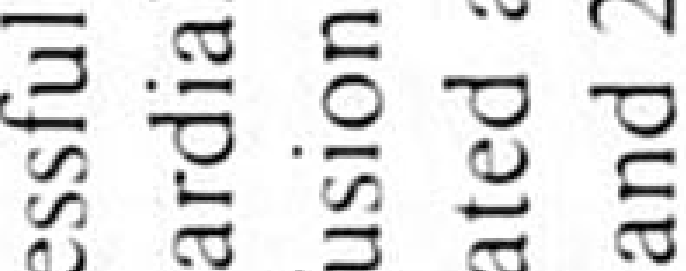

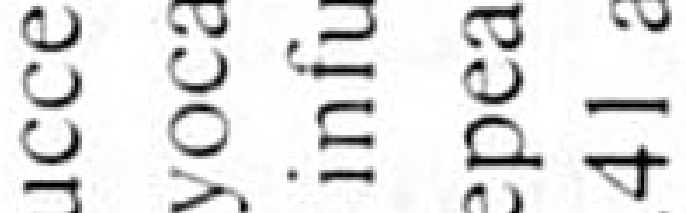

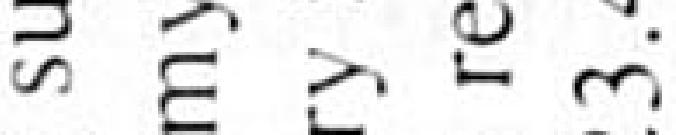

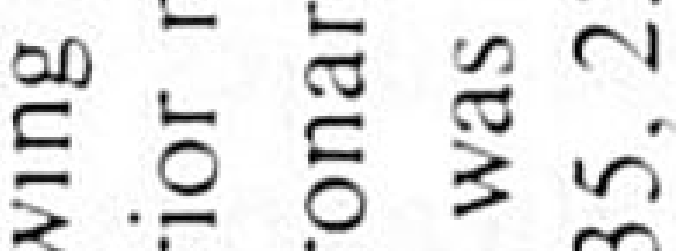

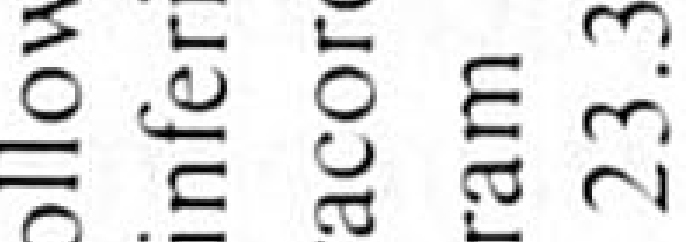
政元

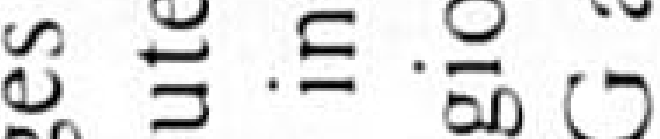

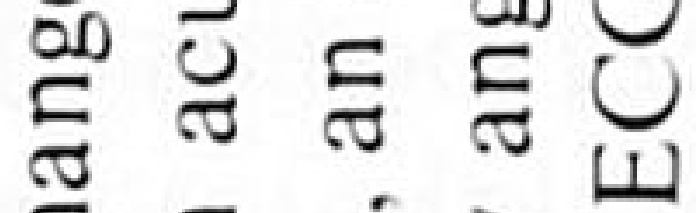
ป

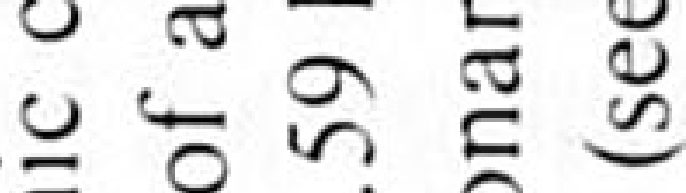

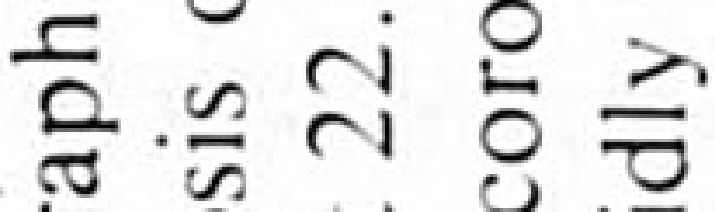

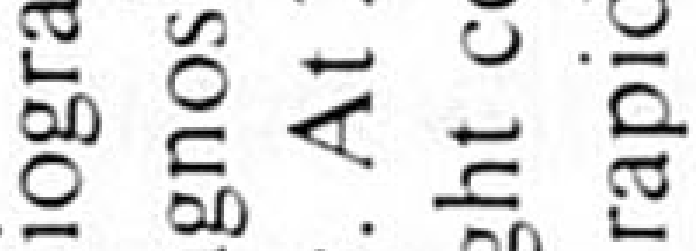

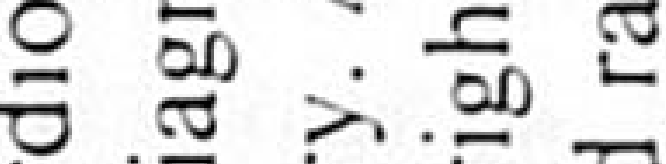

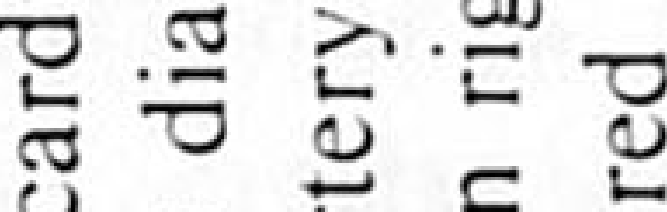
은 흔 记 㐫

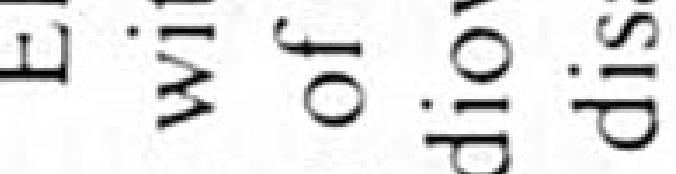
以

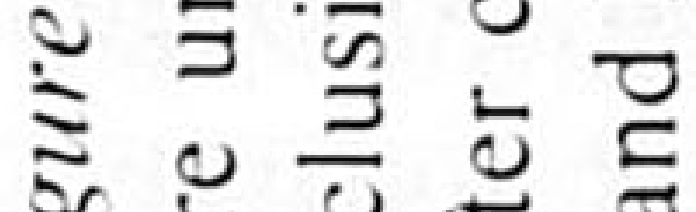

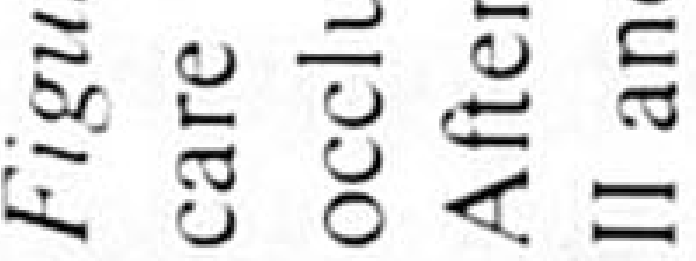


after reperfusion of the ischemic myocardium. During this phase, 14 complications occurred: one ventricular fibrillation, four sinus bradycardias, two cases with severe hypotension, two ventricular tachycardias, two total atrioventricular blocks and two episodes of atrial fibrillation. The details of these complications and the circumstances under which they appeared have made us consider a certain number of preventive measures which will be presented in the discussion.

\section{Discussion}

Any new therapeutic approach to myocardial infarction in order to restore blood flow in the acute phase is a challenge to the clinical investigator. Yet, investigations before intervention are timeconsuming and might postpone the actual onset of recanalization thereby reducing the possible benefits of early reperfusion. Thus there exists a conflict between the time-consuming demands for a critical evaluation and the urgency of the therapeutic intervention. These conflicting factors burdened the first two phases of our study in particular. As a consequence, it was decided that the evaluation of the left ventricular function by contrast ventriculography before and immediately after fibrinolysis was not worth the risk. Other workers have also demonstrated that changes in left ventricular function, secondary to recanalization are unlikely to be present early and that they are best studied several weeks after recanalization ${ }^{[3,4]}$.

What is the maximum delay permissible between the onset of symptoms and the start of reperfusion? A clear answer to this question was given by our initial experience even before a specific time limit was imposed, as the great majority of the failures of recanalization were observed after a 4 - $h$ interval. In the experience of Rentrop ${ }^{[5]}$, the benefit of recanalization is much lower after $3 \mathrm{~h}$, although in patients that present themselves after $6 \mathrm{~h}$, still with chest pain, an attempt to recanalize seems justified, since he has shown that these patients have often well developed collateral vessels and then it still seems possible to salvage ischemic, but viable, myocardium. In the beginning of our experience recanalization was successful in only $62 \%$ but this success rate has subsequently increased to $83 \%$. A rather constant feature was, that in $15 \%$ of our cases no obstructed coronary branches were apparent in the vessels supplying the myocardium suspected to be ischemic from the electrocardiogram. This percentage is comparable to that of other series ${ }^{[6-8]}$.
The five deaths during attempts at recanalization pose several questions. Of the two patients who were in cardiogenic shock at the time of catheterization, the severe hypotension of one was probably induced by left ventriculography and coronary arteriography of the non-obstructed vessels. In the second case this argument is not relevant as ventriculography or contralateral coronary arteriography was not performed before the intracoronary perfusion with streptokinase. During the pilot studies, there were, however, two other patients in cardiogenic shock who were recanalized and apparently stabilized hemodynamically by the successful recanalization. Even so, two additional criteria were developed for admission to the randomized study. These are, the presence of a systolic blood pressure of $90 \mathrm{~mm} \mathrm{Hg}$ and the absence of sinus tachycardia above 100 beats $/ \mathrm{min}$. Patients presenting with cardiogenic shock remain candidates for intracoronary fibrinolysis, but we think that these patients must first be stabilized by means of the intra-aortic balloon pump and they should be evaluated outside the currently accepted protocol. The reason for the death of two further patients is without doubt iatrogenic dislocation of the proximal tail of the thrombus by the tip of the catheter from the left anterior descending artery towards the circumflex artery. In fact, the post mortem in one of our two patients confirms this hypothesis. The peculiar anatomy of the coronary vessels must be stressed in these two patients, as a very short main stem artery was present together with a very proximal thrombosis of the left anterior descending artery. The existence of a tail of thrombus proximal to the initial site of the thrombosis has been observed and confirmed during post mortem in other patients with a myocardial infarction in whom an intravenous injection of labeled fibrinogen was performed before death[9]. In the last patient, myocardial rupture is strongly suspected, but no post mortem was performed, so that this case, like others reported by Hugenholtz and Rentrop ${ }^{[2]}$, must provide another cause for worry since the thrombolytic therapy could have induced the rupture. The non-fatal complications such as arrhythmias and conduction disturbances, which are inherent in the acute phase of myocardial infarction, cannot be attributed solely to the fibrinolytic intervention or the angiographic investigation. In fact, $21 \%$ of these complications were observed in the catheterization laboratory before the catheterization procedure was started. Pantridge et al. ${ }^{[10]}$ have recently published new data 
on arrhythmias in the first hours of acute myocardial infarction. Of the 294 patients seen within $1 \mathrm{~h}$ of onset of acute myocardial infarction, bradyarrhythmia occurred in $34 \%$ within the first hour and was most frequent among those with inferior infarction. Ventricular fibrillation occurred in 55 $(19 \%)$ patients, developing in $41(75 \%)$ within $2 \mathrm{~h}$ and $46(84 \%)$ within $4 \mathrm{~h}$ of the onset of chest pain. Systolic blood pressure at initial examination was $80 \mathrm{~mm} \mathrm{Hg}$ or less in nearly $25 \%$ of the patients. Similar data have been observed in our unit ${ }^{[11]}$. Although possibly not related, it remains possible that a high percentage (37\%) of complications were associated with the intracoronary, intraventricular or intra-aortic injection of radio-opaque contrast medium, the high ionic load of which is known to cause arrhythmias. Consequently, three further preventive measures have been taken: omission of the left ventriculography before an attempt at recanalization; utilization of a non-ionic contrast medium, and severe restriction of the amounts of contrast used during the diagnostic and therapeutic phase of intracoronary fibrinolysis.

In addition, we feel that the angiographer must refrain from trying to obtain spectacular coronary angiographic 'images', since this necessitates the injection of large amounts of contrast medium. The cardiodepressant effect of urographin $76 \%$ particularly in ischemic and prenecrotic regions, was recently emphasized. On the other hand, the newer non-ionic contrast media alter the coronary sinus blood chemistry less, even with critical coronary artery stenosis ${ }^{[2]}$. Yet, in the entire procedure the most critical time remains the first coronary angiogram after recanalization and reperfusion of the ischemic myocardium since $20 \%$ of our avoidable complications have occurred at that time. Arrhythmias and conduction disturbances have also been shown to be associated with the reperfusion itself and with the migration of the dissolving thrombus and its particles. The percentage of complications observed during the perfusion of the fibrinolytic agent itself is $26 \%$. Here the rhythm disturbances observed are mainly uniform and multiform ventricular premature complexes, occasionally associated with doublets and bigeminy. This corresponds to observations made in animal experiments where reperfusion was sudden[13,14]. Ganz and coworkers have therefore suggested the use of intracoronary lidocaine for controlling severe and repetitive rhythm disturbances ${ }^{[15]}$. In keeping with our own experimental data, we would add to these recommendations that of infusing nifedipine. This car- dioprotective agent proves to be antiarrhythmic under these circumstances ${ }^{[16]}$. One last point to be stressed is the very high incidence of complications during successful recanalization of the right coronary artery. Only the anatomy of the right coronary artery, which supplies the atrioventricular nodal branch, seems to be able to explain the high incidence of rhythm and conduction disturbances during recanalization of this artery.

In summary, the present data stress the inherent risks of intracoronary fibrinolysis. Some of these can be eliminated by several supportive measures or by a more cautious approach of performing angiography than currently used in most laboratories. As long as the short- and long-term benefits of therapeutic intervention are not clearly demonstrated, it remains possible that the risks inherent to the intervention will exceed an acceptable level.

\section{References}

[1] Muller JE, Stone PH, Moskis JE, Braunwald E. Let's not let the genie escape from the bottle again. N Eng J Med 1981; 304: 1294-6.

[2] Hugenholtz PG, Rentrop P. Thrombolytic therapy for acute myocardial infarction: quo vadis? A review of the recent literature. Eur Heart J 1982; 3: 395-403.

[3] Reduto LA, Smalling RW, Freund GC, Gould KL. Intracoronary infusion of streptokinase in patients with acute myocardial infarction: effects of reperfusion on left ventricular performance. Am J Cardiol 1981; 48: 403-9.

[4] Théroux P, Ross J, Franklin D, Kemper WS, Sasayama S. Coronary arterial reperfusion, III. Early and late effects on regional myocardial function and dimensions in consious dogs. Am J Cardiol 1976; 38 : 599-606.

[5] Rentrop P, Merx W, Mathey D, Blanke H, Rutsch W, Karsch KR. Functional results of streptokinasereperfusion in relation to collaterals and duration of symptoms. Circulation 1981; 64(suppl IV): 194. (abstract).

[6] DeWood MA, Spores J, Notske R, Mouser LT, Burroughs R, Golden MS, Lang HT. Prevalence of total coronary occlusion during the early hours of transmural myocardial infarction. $N$ Engl $J$ Med 1980; 303: 897-902.

[7] Merx W, Dorr R, Rentrop P, et al. Evaluation of the effectiveness of intracoronary streptokinase infusion in acute myocardial infarction: postprocedure management and hospital course in 204 patients. Am Heart J 1981; 102: 1181-7.

[8] Lee G, Amsterdam EA, Low R, Joye JA, Kimchi A, Demaria AN, Mason DT. Efficacy of percutaneous transluminal coronary recanalization utilizing streptokinase thrombolysis in patients with acute myocardial infarction. Am Heart J 1981: 102: 1159-67.

[9] Fulton WFM, Sumner DJ. Radiofibrinogen in a study of fatal mycardial infarction. Thromb Haemostas 1979; 43: 38. (Abstract). 
[10] Pantridge JF, Webb SW, Adgey AAJ. Arrhythmias in first hours of acute myocardial infarction. Progr Cardiovasc Dis 1981; 23: 265-78.

[11] Wolffenbuttel BHR, Verdouw PD, Hugenholtz PG. Immediate and two year prognosis after acute myocardial infarction: prediction from non-invasive as well as invasive parameters in the same individual. Eur Heart J 1981; 2: 375-87.

[12] Higgins C, Gerber K, Yuh YS, Koziol J. Regional myocardial hemodynamic and metabolic effects of ionic and non ionic contrast media in the normal and ischemic state. Circulation 1981; 64 (suppl IV): 1340. (Abstract).

[13] Verdouw PD, Hartog JM, ten Cate FJ, et al. Effects of nifedipine on the recovery of regional myocardial performance during reperfusion of ischemic myocardium. Progr Pharmacol 1981; 4/2: 91-100.

[14] Henry PD, Schuchleib R, Borda LJ, Roberts R, Williamson JR, Sobel B. Effects of nifedipine on myocardial perfusion and ischemic injury in dogs. Circ Res 1978; 43: 372-80.

[15] Peter T, Mercier JC, Lando V, Kanmatsuse K, Ganz W. Successful prevention and treatment of reperfusion ventricular tachycardia with intracoronary lidocaine. Circulation 1981; 64 (suppl IV): 319.

[16] Verdouw PD, ten Cate FJ, Hartog HM, Hugenholtz PG. Does nifedipine protect against reperfusion damage? Am J Cardiol 1981; 47: 461. (Abstract). 1 